\title{
Esquema de Diferenças Finitas Otimizado Aplicado a Migração Reversa no Tempo em Meios com Isotropia Transversalmente Vertical
}

\author{
Borges $^{2^{*}}$, I. F.; Silva ${ }^{1}$, B. S..; Barbosa ${ }^{2}$, C. H. S.; Vidal ${ }^{2}$, A. D.; Landau', L.
}

(1. UFRJ/COPPE/LAMCE/Lab2M - Laboratório Multidisciplinar de Modelagem \& 2. PETREC - Petroleum Research and

Technology)

Copyright 2016, SBGf - Sociedade Brasileira de Geofísica

Este texto foi preparado para a apresentação no VII Simpósio Brasileiro de Geofísica, Ouro Preto, 25a 27 de outubro de 2016. Seu conteúdo foi revisado pelo Comitê Técnico do VIISimBGf, mas não necessariamente representa a opinião da SBGf ou de seus associados. E proibida a reprodução total ou parcial deste material para propósitos comerciais sem prévia autorização da SBGf.

\section{Resumo}

A Migração Reversa no Tempo (RTM, do inglês, Reverse Time Migration) exibe grandes vantagens sobre outros métodos de imageamento, uma vez que essa técnica é baseada na solução numérica da equação completa da onda. A anisotropia sísmica é uma propriedade importante que pode ser observada em muitas áreas de exploração (por exemplo, Mar do Norte e Golfo do México). Assim, ela deve ser considerada no fluxo de processamento sísmico para poder alcançar melhores resultados. A fim de obter uma redução de dispersão na modelagem empregada na RTM em meios VTI (do inglês, Vertical Transversely Isotropic), neste trabalho aplicamos um esquema de diferenças finitas otimizado onde utiliza-se uma janela binominal com o intuito de obter um operador de Diferenças Finitas mais preciso. Com tal precisão foi utilizado um espaçamento entre pontos da malha maior do que o utilizado quando aplicase o operador de Diferenças Finitas convencional (Taylor).

\section{Introdução}

A aquisição sísmica consiste em emitir uma onda mecânica na direção da subsuperfície e registrar, através dos sensores (geofones ou hidrofones), o tempo de chegada $e$ as amplitudes das energias refletidas e refratadas nas interfaces geológicas. Após a aquisição dos dados sísmicos, a migração sísmica surge como uma sequência natural na etapa do processamento (Yilmaz, 2001).

A migração tem o objetivo de transformar os campos de ondas registrados na superfície ou no fundo oceânico, em uma imagem que represente a estrutura geológica (seção migrada).

A Migração Reversa no Tempo (RTM, do inglês, Reverse Time Migration), proposta por Baysal (1983), é um método que utiliza a Equação Completa da Onda, não apresenta limitações quanto à variação lateral de velocidade e não impõe limitação quanto aos mergulhos das camadas. Na RTM, ocorre a propagação reversa do sinal registrado na superfície. Durante essa propagação, aplica-se uma determinada condição de imagem.

A propagação reversa da onda é realizada utilizando Equação Acústica da Onda, que normalmente é solucionada pelo Método das Diferenças Finitas (MDF). O MDF é um dos métodos numéricos mais populares, por ser de fácil implementação e também um dos mais bem sucedidos por ser ideal para modelos complexos (Alford et al., 1974).

A fim de melhorar a precisão do MDF Chu \& Stoffa (2012) encontraram duas famílias de Binomial Window que podem ser utilizadas para derivar os operadores de Diferenças Finitas (DF) analiticamente. Zhang \& Yao (2013) reduziram as dispersões numéricas do MDF, na presença de componentes de altas frequências.

Dentro deste contexto, o presente trabalho tem como objetivo apresentar a aplicação dos coeficientes de Diferenças Finitas Otimizados para a obtenção da solução numérica da equação pseudo-acústica da onda. Esta será utilizada para realizar o imageamento do conjunto de dados BP 2007 com a técnica RTM VTI.

\section{Teoria}

Imageamento sísmico é um conjunto de procedimentos, que objetiva transformar o campo de ondas registrado em uma imagem que representará as estruturas geológicas. A Migração Reversa no Tempo é uma técnica de imageamento sísmico que propaga o campo da fonte e o campo registrado na posição dos receptores 
reversamente no tempo utilizando a equação completa da onda (Baysalet al., 1983; McMechan, 1983; Whitmore, 1983).

O campo da fonte $S(\vec{r}, t)$ e o campo do receptor $R(\vec{r}, t)$ podem ser utilizados para satisfazer a condição de imagem, que é essencial para formar a imagem migrada. A condição de imagem usada foi à correlação cruzada como escrita abaixo:

$$
I(\vec{r}, t)=\int_{0}^{t_{f}} S(\vec{r}, t) R(\vec{r}, t) d t
$$

onde $t_{f}$ é o tempo total da aquisição, $t$ é a variável temporal e $\vec{r}$ um vetor, que representa as coordenadas espaciais $\vec{r}=(x, y, z)$.

Para calcular o campo de onda da fonte e do receptor, nas próximas seções, a equação pseudo-acústica da onda para meios com isotropia transversalmente vertical será exposta.

\section{Operador de Diferenças Finitas}

Uma forma de reduzir a dispersão numérica, é a adoção de funções janelas para gerar coeficientes otimizados para os operadores de Diferenças Finitas. Chu e Stoffa (2012) propuseram uma janela $w_{n}$ para obter coeficientes de DF otimizado, que podem ser gerados da seguinte forma:

$$
\frac{\partial^{2} f}{\partial x^{2}} \approx \frac{1}{\Delta \mathrm{x}^{2}} \sum_{\mathrm{n}=-\frac{\mathrm{N}}{2}}^{\frac{\mathrm{N}}{2}} \mathrm{w}_{\mathrm{n}}\left[-\frac{2}{\mathrm{n}^{2}} \cos (\mathrm{n} \pi)\right] \mathrm{f}(\Delta \mathrm{x}),
$$

onde $w_{n}$ é gerado a partir da seguinte função janela otimizada:

$$
w_{n}=\frac{\left(\begin{array}{c}
N+M \\
\frac{N+M}{2}+n
\end{array}\right)}{\left(\begin{array}{c}
N+M \\
\frac{N+M}{2}
\end{array}\right)} .
$$

Utilizando uma forma final para o operador de DF otimizado, pode-se combinar a função janela $w_{n}$ com todas as partes da Equação 2 obtendo:

$$
\frac{\partial^{2} f}{\partial x^{2}} \approx \frac{1}{\Delta \mathrm{x}^{2}} \sum_{\mathrm{n}=-\frac{\mathrm{N}}{2}}^{\frac{\mathrm{N}}{2}} \mathrm{c}_{\mathrm{n}} \mathrm{f}(\Delta \mathrm{x})
$$

onde $c_{n}$ é a forma final dos coeficientes a serem utilizados na implementação, e pode ser definido como:

Coeficientes Otimizado- Binominal Window:

$$
\begin{aligned}
& c_{n}=w_{n}\left[-\frac{2}{\mathrm{n}^{2}} \cos (\mathrm{n} \pi)\right], \\
& c_{0}=\sum_{\mathrm{n}=-\mathrm{N} / 2}^{\mathrm{N} / 2} \mathrm{c}_{-\mathrm{n}}+\mathrm{c}_{\mathrm{n}} .
\end{aligned}
$$

Considerando $\quad M=0$ temos os coeficientes convencionais de Taylor. O parâmetro $M$ dessa função janela é difícil de determinar e ao mesmo tempo necessita de ser manuseado com cautela, pois pode afetar significativamente o resultado final. Zhang e Yao (2013) determinaram os coeficientes $c_{n}$ utilizando um esquema de otimização que utiliza a maximização da convergência do número de onda, dado uma limitação do erro. Foi examinado o pico do erro entre o operador otimizado no domínio do número de onda e no domínio do número de onda analítico. Para gerar os coeficientes otimizados, foi usado o algoritmo de minimização Simulated Annealing (SA). Tais coeficientes são utilizados neste trabalho.

\section{Análise do Erro Absoluto}

Com intuito de avaliar a melhoria na propriedade de dispersão, foi construído o gráfico da análise do erro espectral da derivada segunda para diferentes ordens de precisão (Figura 1).

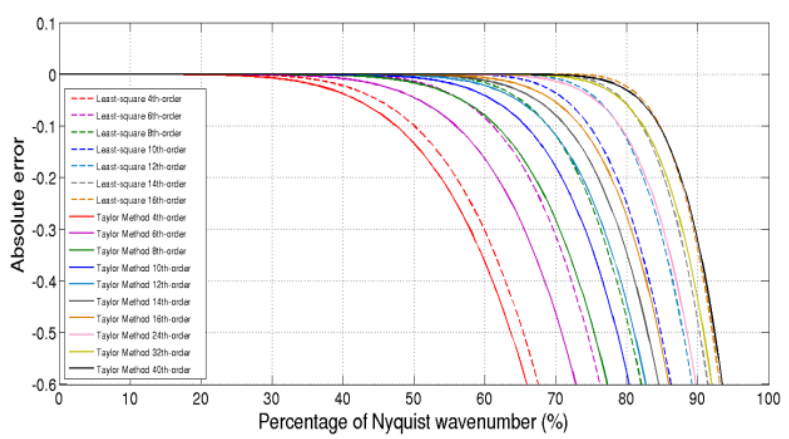

Figura 1- Análise do Erro Espectral para o Operador da Derivada Espacial de Segunda Ordem (Silva et al., 2014). 
Para isso, utilizou-se a fórmula do erro espectral absoluto, escrita em função do número de onda normalizado pelo número de onda de Nyquist, ou seja:

$$
\operatorname{Erro}\left(\frac{K_{x}}{K_{x n}}\right)=-\left[c_{0}+2 \sum_{n=1}^{\frac{N}{2}} c_{n} \cos \left(\frac{k_{x}}{k_{x n}} n \pi\right)\right]-\left(\frac{k_{x}}{k_{x n}} \pi\right) \text {. }
$$

\section{Equação acústica da onda em meios VTI}

As equações acústicas anisotrópicas são geralmente deduzidas da relação de dispersão (Alkhalifah, 1997; Zhouet al., 2006, Du et al., 2008). Du et al.(2008) propôs uma equação acoplada de segunda ordem. Tal equação para meios VTI 2D é dada por:

$$
\begin{aligned}
& \frac{\partial^{2} p}{\partial t^{2}}=v_{p x}^{2} \frac{\partial^{2} p}{\partial x^{2}}+v_{p z}^{2} \frac{\partial^{2} q}{\partial z^{2}}, \\
& \frac{\partial^{2} q}{\partial t^{2}}=v_{p n}^{2} \frac{\partial^{2} p}{\partial x^{2}}+v_{p z}^{2} \frac{\partial^{2} q}{\partial z^{2}},
\end{aligned}
$$

onde $p$ e $q$ são as funções campo de onda e campo auxilia, respectivamente; $v_{p z}$ é a velocidade vertical da onda $\mathrm{qP} ; \quad v_{p n}=v_{p z} \sqrt{1+2 \delta}$ é a velocidade normal moveout (NMO) da onda $\mathrm{qP} ; v_{p x}=v_{p z} \sqrt{1+2 \varepsilon}$ é a velocidade horizontal da onda $\mathrm{qP}$; e $\varepsilon$ e $\delta$ são os parâmetros anisotrópicos definidos por Thomsen (1986).

A discretizacão das derivadas das equações $7 \mathrm{a}$ e $7 \mathrm{~b}$ geralmente são realizadas com o esquema de diferenças finitas. Uma discretizacão de segunda ordem é realizada nas derivadas temporais como segue:

$$
\begin{aligned}
& \frac{\partial^{2} p}{\partial t^{2}} \approx \frac{1}{\Delta t^{2}}\left[-2 p_{0,0,0}^{0}+\left(p_{0,0,0}^{-1}+p_{0,0,0}^{1}\right),\right. \\
& \frac{\partial^{2} q}{\partial t^{2}} \approx \frac{1}{\Delta t^{2}}\left[-2 q_{0,0,0}^{0}+\left(q_{0,0,0}^{-1}+q_{0,0,0}^{1}\right),\right.
\end{aligned}
$$

As altas ordens de discretização são utilizadas nas derivadas espaciais:

$$
\begin{aligned}
& \frac{\partial^{2} p}{\partial x^{2}} \approx \frac{1}{h^{2}}\left[c_{0} p_{0,0,0}^{0}+\sum_{n=1}^{\frac{N}{2}} c_{n}\left(p_{-n, 0,0}^{0}+p_{n, 0,0}^{0}\right)\right], \\
& \frac{\partial^{2} q}{\partial z^{2}} \approx \frac{1}{h^{2}}\left[c_{0} q_{0,0,0}^{0}+\sum_{n=1}^{\frac{N}{2}} c_{n}\left(q_{0,0,-n}^{0}+q_{0,0, n}^{0}\right)\right],
\end{aligned}
$$

sendo $N$ a ordem do operador de DF, $c_{m}$ são os coeficientes de cada ponto do stencil, $\Delta t$ é o passo de tempo e $h$ o espaçamento da malha.

\section{Resultados}

Nós utilizamos os stencils $8 \mathrm{a}, 8 \mathrm{~b}, 9 \mathrm{a}$ e $9 \mathrm{~b}$ para resolver as equações $7 \mathrm{a}$ e $7 \mathrm{~b}$. Os coeficientes de DF são determinados pelas equações $5 \mathrm{a}$ e $5 \mathrm{~b}$. $\mathrm{O}$ primeiro exemplo é uma modelagem em um modelo 2D homogêneo com anisotropia elíptica. Os parâmetros referente a modelagem são dados por: $V_{p z}=3000 \mathrm{~m} / \mathrm{s}$, $\epsilon=0.24, \delta=0.24, d t=0.4 \mathrm{~ms}, h=24 \mathrm{~m}, N=6$, onde a quantidade de pontos na malha de DF é $300 \times 300$.

A Figura 1 mostra o snapshot da modelagem utilizando o método convencional (Figura 2a) e método otimizado (Figura 2b). Pode-se observar que a modelagem otimizada apresentou melhor propriedade de dispersão que a convencional.

A Figura 3 apresenta 0 registro de um traço sísmico realizado em $[x, z]=[3600,1440]$ metros. A Figura 3a representa o resultado do registro referente a modelagem utilizando o operador convencional e da Figura $3 \mathrm{~b}$ o registro do operador otimizado. Observa-se novamente que a modelagem otimizada apresentou menor dispersão que a convencional.

A RTM VTI utiliza em seu núcleo a equação pseudoacústica para meios com isotropia transversamente vertical. Com isso, pode-se alcançar uma melhor precisão ao utilizar os operadores otimizados para obter a solução da equação da onda. O resutado da aplicação desta técnica na migração pode ser observado na Figura 4.

\section{Discussões e Conclusões}

O stencil de Diferenças Finitas foi utilizado para aproximar as derivadas espaciais das equações $7 a$ e $7 b$. Os coeficientes de Taylor gerados pela Binomial Window 
$(M=0)$ foram inseridos na discretização da equação pseudo-acústica VTI proposta por Du et al. (2008) e comparados com os provenientes da função janela otimizada. Os resultados das Figura 2 e 3 demonstraram que os coeficientes otimizados foram mais precisos. Tal precisão foi alcançada com 0 mesmo custo computacional do método convencional.

Após melhorar a precisão do cálculo das derivadas da equação da onda com os operadores otimizados, esta técnica foi herdada para realizar a RTM do conjunto de dados do Benchmark BP2007 2D.

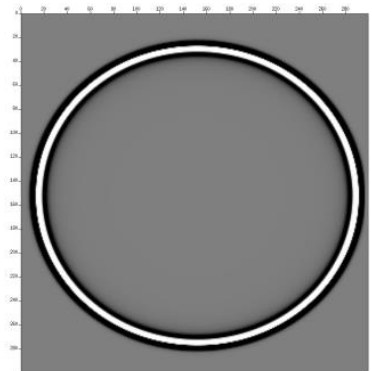

(a) Modelagem Convencional

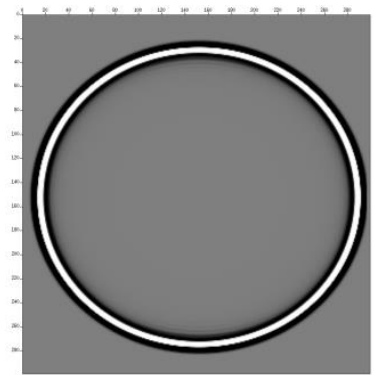

(b) Modelagem Otimizada
Figure 2: Snapshots da modelagem gravado em xxx s pelos método: (a) Convencional e (b) Otimizado, sobre um modelo 2D homogêneo, pseudo-acústico com anisotropia elíptica. A malha de DF possui $300 \times 300$ pontos. A fonte utilizada foi a Ricker, com freqüência de $40 \mathrm{~Hz}$ e posicionada no centro do modelo.
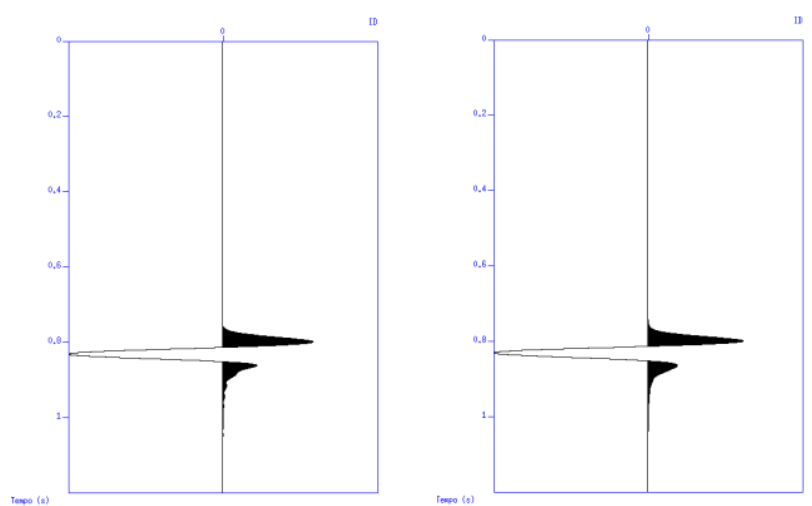

Figure 3: Traço sísmico registrado da Figura 1 pelos métodos: (a) Convencional and (b) Otimizado. O receptor foi posicionado em $[x, z]=[3600,1440]$ metros.

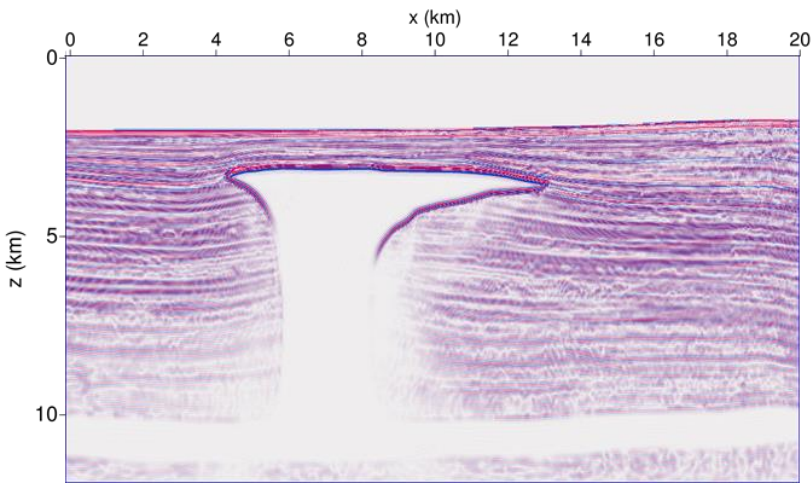

Figure 4: Seção sísmica migrada com a técnica RTM VTI.

\section{Agradecimentos}

Os autores gostariam de agradecer a BP por disponibilizar o conjunto de dados BP 2007 2D. Gostariam também de agradecer ao Laboratório de Métodos Computacionaos em Engenharia da COPPE/UFRJ e a PETREC - Petroleum Research and Technology pelo suporte financeiro e técnico. Finalmente, ao Dr. Josias Silva pelas contribuições no desenvolvimento do trabalho.

\section{Referências}

ALFORD, R. M., KELLY, K. R., BOORE, D. M., 1974, "Accuracy of finite difference modeling of the acoustic wave equation", Geophysics, v. 39, n. 6, pp. 834-842.

ALKHALIFAH, T., 1997. An acoustic wave equation for anisotropic media. Stanford Exploration Project, v. 95, pp. 283-308.

BAYSAL, E., KOSLOFF, D. D., SHERWOOD, J. W. C., 1983, "Reverse Time Migration", Geophysics, v. 48, pp. 1514-1524.

CHU, C., STOFFA, P. L., 2012, "Determination of finitedifference weights using scaled binomial windows", Geophysics, v. 77, pp. 57-67.

DU, X., FLETCHER, R.P., and FOWLER, P. J., 2008.A new pseudo-acoustic wave equation for VTI media. $70^{\text {th }}$ EAGE Conference \& Exhibition - Rome, Italy. 
LIU, Y., SEN, Y. M., 2009, "A new time-space domain high-order finite-difference method for the acoustic wave equation", Journal of Computacional Physics, v. 228, pp. 8779-8806.

LIU, Y., 2013, "Globally optimal finite-difference schemes based on least squares", Geophysics, v. 78, pp. T113T132.

MCMECHAN, G. A., 1983, "Migration by extrapolation of time-dependent boundary values", Geophysical Prospecting, v. 31, pp. 413-420.

SILVA, B. S. ; BARBOSA, C. H. S. ; CONTRERAS, C. S. R. ; SILVA, J. J.; LANDAU, L. . "Optimized Scheme of Finite Differences Applied to VTI Acoustic Modeling". In: 16 IWSA International Workshop on Seismic Anisotropy, 2014, Natal, 2014.

THOMSEN, L., 1986. Weak elastic anisotropic: Geophysics, v. 51, pp. 1954-1966.

WHITMORE, N. D., 1983, "Iterative depth migration by backward time propagation”, SEG 53rd Annual International Meeting, pp. 827-830.

YILMAZ, O., 2001, Seismic Data Analysis. Tulsa, Society of Exploration Geophysicits.

ZHANG, J. H., YAO, Z. X., 2013. Optimized finitedifference operator for broad-band seismic wave modeling: Geophysics, v.78, pp. 1-6.

ZHOU, H., ZANG, G., and BLOOR, R., 2006. An anisotropic wave equation for VTI media.68th EAGE Conference \& Exhibition - Vienna, Austria. 